\title{
Limited Feedback Multiuser MISO Systems with Differential Codebooks in Correlated Channels
}

\author{
Jawad Mirza*, Pawel A. Dmochowski*, Peter J. Smith ${ }^{\dagger}$ and Mansoor Shafi ${ }^{\ddagger}$ \\ * School of Engineering and Computer Science, Victoria University of Wellington, New Zealand \\ $\dagger$ Department of Electrical and Computer Engineering, University of Canterbury, New Zealand \\ $\ddagger$ Telecom New Zealand, New Zealand \\ Email:\{jawad.mirza, pawel.dmochowski\}@ecs.vuw.ac.nz, p.smith@elec.canterbury.ac.nz,mansoor.shafi@telecom.co.nz
}

\begin{abstract}
In this paper, we present a differential codebook design by modifying the Grassmannian codebook for a singlecell multiuser (MU) multiple-input single-output (MISO) system operating under spatially and temporally correlated channels. The differential codebook design involves scaling and rotation methods that help a codebook to track the slow varying channel. We propose an adaptive scaling technique that improves the performance of the system in correlated channels without any additional feedback information. Monte Carlo simulations show that the proposed differential codebook reduces quantization errors and improves sum-rate performance as compared to other differential codebooks designed for spatially and temporally correlated MISO channels.
\end{abstract}

\section{INTRODUCTION}

Multiple-input multiple-output (MIMO) has shown to be a promising technology that meets the demand of high data rates in $4 \mathrm{G}$ and beyond wireless communication systems [1]. Channel state information (CSI) at the transmitter helps to improve the performance of MIMO systems by enabling the transmitter to exploit the channel conditions [2]. In MIMO frequency division duplexing (FDD) systems, it is not possible for a transmitter to acquire a CSI without any feedback information from a receiver [3]. For this purpose, a codebook with the same entries and size is maintained at both transmitter and receiver, containing quantized channel information. Due to bandwidth limitations, instead of feeding back the entire channel information to the transmitter, the receiver feeds back the index of the nearest codebook entry (or codeword) to the transmitter via a low-rate feedback link. The number of bits required to send the index of the selected codeword to the transmitter is considered as feedback overhead.

One of the major factors influencing codebook design in limited feedback systems is the propagation environment. Depending on a level of spatial and temporal correlation, a codebook can be designed accordingly. In codebook-based precoding, research on designing a fixed codebook for independent and identically distributed (i.i.d.) channels has dominated for many years. Recently, the research focus has shifted towards adaptive codebook precoding, where the codebook tracks the correlated channel and updates itself accordingly. This approach is driven by the fact that MIMO real-world channels are both spatially and temporally correlated and codebooks designed for i.i.d. channels do not perform well in correlated channels [4], [5].
Next generation wireless systems are employing both adaptive and non-adaptive codebook-based precoding. In the IEEE $802.16 \mathrm{~m}$ standard, three types of codebooks are used; base codebook, adaptive (or transformation) codebook and differential codebook [6]. The base codebook is a fixed codebook that does not vary with the changing channel. The adaptive codebook requires the feedback of a long term transmit correlation matrix and the differential codebook exploits the temporal correlation present in the channel and updates the codebook accordingly [7]. In order to minimize errors, the differential process has to be periodically reset [8]. Third Generation Partnership Project (3GPP) is engaged in looking at optimising codebooks for use in LTE advanced with an aim to select a set of codebooks that may be chosen to ensure high performance over a range of conditions [9]. The design of these codebooks are based on DFT, Grassmannian and Householder matrix codebooks [10]-[14]. The key conclusion from a large number of studies is that there is no single codebook that performs best over all types of scenarios including fading, mobile speed, antenna layout etc. Therefore, a subset of codebooks need to be considered, so that the handset may select a codebook according to the conditions it experiences.

In spatially correlated channels, the idea of rotating the codebook in the direction of the transmit correlation is studied in [4]. Grassmannian beamforming codebook [2] is used in [4] and spatial correlation is modeled by a Kronecker model with no temporal correlation. This rotated Grassmannian codebook is shown to outperform the stationary Grassmannian codebook. A systematic codebook design has been developed in [15], where the beamforming codebook is both scaled and rotated in the direction of transmit correlation. This systematic codebook performs well under Kronecker channels. In temporally correlated channels, the differential precoding technique is studied in [16], where the selected codebook entry is multiplied by the previous precoding matrix in order to obtain the current precoding matrix. This method reduces the feedback overhead by 1-2 bits as compared to the Grassmannian codebook in MIMO systems with four transmit and two receive antennas. Rotation-based differential codebooks are investigated in [17], [18], where codebooks consist of a set of rotation matrices. The differential codebook methodology employed by IEEE $802.16 \mathrm{~m}$ is explained in [19]. A polar-cap differential codebook design for a multiuser MISO channel is presented in [20] 
for temporally and spatially correlated channels. The polar-cap differential structure is obtained by scaling a fixed Grassmannian codebook such that one codeword is at the center and all other codewords are on the radius of the polar-cap differential codebook. This codebook is then rotated such that the selected codeword becomes a new center for the codebook in the next feedback period. This polar-cap differential codebook outperforms the random vector quantization (RVQ) [21] and rotation-based differential [18] codebooks for the same number of feedback bits.

In this paper, a differential codebook for the MU MISO system is proposed, where the codewords of the Grassmannian codebook are scaled such that one codeword is at the center and all other codewords lie inside the radius of the differential codebook. This differential codebook is then rotated to a selected codeword after each feedback interval. Both scaling and rotation methods have a significant effect on the performance of the differential codebook. The polar-cap differential codebook [20] is used as a baseline model for the performance assessment of our proposed differential codebook. The main contributions of this paper are:

- We propose a differential codebook design for a MU MISO system in spatially and temporally correlated channels. The differential codebook design is based on the Grassmannian codebook, modified by performing scaling and rotation operations. The scaling method allows us to distribute the codewords evenly inside the radius of differential codebook, thus, reducing quantization errors.

- We propose an adaptive scaling method for the differential codebook based on current and previous selected precoding vectors. This scaling does not require any additional feedback, is shown to improve the performance significantly compared to the fixed scaling at low speeds.

- We introduce a new SVD-based rotation method for codebook rotation that effectively rotates a single- or multi-stream codebook to any desired location ${ }^{1}$.

This paper is organised as follows. In Section II, the description of the system model is presented. In Section III, we give a detailed explaination of the proposed differential codebook design and procedure. Section IV discusses various operations performed on differential codebook. Monte Carlo simulation results and conclusions are presented in Sections $\mathrm{V}$ and VI respectively.

\section{SySTEM MODEL}

Consider a single-cell MU limited feedback MISO system that consists of $K$ users supported by a single base-station (BS) with $n_{\mathrm{T}}$ transmit antennas. Each user has one recieve antenna $\left(n_{\mathrm{R}}=1\right)$ and all users are scheduled simultaneously. The total number of users is equal to the number of transmit antennas, $K=n_{\mathrm{T}}$. At time instance $t$, the received signal of the $i^{\text {th }}$ user is given by

\footnotetext{
${ }^{1}$ Due to space constraints, the differential codebook for MU MIMO systems with multi-stream transmission is deferred to the journal version of this paper.
}

$$
r_{t, i}=\sqrt{\frac{\rho}{K}} \mathbf{h}_{t, i}^{\dagger} \mathbf{x}_{t}+n_{t, i},
$$

where $\rho$ is the signal-to-noise ratio (SNR) of the link, $\mathbf{h}_{t, i}$ represents the $n_{\mathrm{T}} \times 1$ channel vector between the $\mathrm{BS}$ and the $i^{\text {th }}$ user, $n_{t, i}$ is a complex Gaussian $\mathcal{C} \mathcal{N}(0,1)$ noise term, and $\mathbf{x}_{t}$ is the $n_{\mathrm{T}} \times 1$ transmitted signal from the BS. For a temporally correlated scenario, the channel vector, $\mathbf{h}_{t, i}$, for the $i^{\text {th }}$ user is modeled by a first-order Gauss-Markov process

$$
\mathbf{h}_{t, i}=\epsilon \mathbf{h}_{t-1, i}+\sqrt{1-\epsilon^{2}} \mathbf{g}_{t, i},
$$

where $\epsilon$ is a time correlation coefficient defined by the Jakes' model [22], $\epsilon=J_{0}\left(2 \pi f_{D} T\right), f_{D}$ and $T$ are the Doppler frequency and channel instantiation interval respectively, and $\mathbf{g}_{t, i}$ is a $n_{\mathrm{T}} \times 1$ vector of complex Gaussian random variables with zero mean and unit variance. At time $t=0$, the channel vector, $\mathbf{h}_{0, i}$, is independent of $\mathbf{g}_{t, i}$ and the noise term, $n_{t, i}$, is also independent of both $\mathbf{h}_{0, i}$ and $\mathbf{g}_{t, i}$. We assume that all the users experience the same time correlation. In order to allow for a fair comparison of codebook methods, we adopt the spatially and temporally correlated channel model from [20], written as

$$
\mathbf{h}_{t, i}=\epsilon \mathbf{h}_{t-1, i}+\mathbf{R}_{i}^{1 / 2} \sqrt{1-\epsilon^{2}} \mathbf{g}_{t, i}
$$

where $\mathbf{R}_{i}^{1 / 2}$ is the transmit spatial correlation matrix for the $i^{\text {th }}$ user [23] defined by

$$
\mathbf{R}_{i}=\left[\begin{array}{cccc}
1 & r_{i} & \ldots & r_{i}^{\left(n_{t}-1\right)} \\
r_{i}^{\dagger} & 1 & & \\
\vdots & & \ddots & \\
r_{i}^{\left(n_{t}-1\right) \dagger} & & & 1
\end{array}\right] .
$$

In (4), the phase of correlation coefficient, $r_{i}$, for each user is uniformly generated from 0 to $2 \pi$, whereas, the magnitude of the correlation coefficient, $\left|r_{i}\right|$, is the same for all users. We assume that each user maintains a finite size codebook, denoted by $\mathbf{F}_{i}$, that consists of $M=2^{B}$ entries (or codewords). $B$ is the number of bits required to feedback the index of the selected codeword to the transmitter (all users use the same number of bits $B$ ). The codebook for the $i^{\text {th }}$ user can be represented by $\mathbf{F}_{i}=\left[\mathbf{f}_{1, i}, \mathbf{f}_{2, i}, \ldots, \mathbf{f}_{\mathrm{M}, i}\right]$. At time instance $t$, the user $i$ selects that codebook entry which maximizes the received SNR, such that $\hat{\mathbf{f}}_{t, i}=\operatorname{argmax}_{1 \leq j \leq 2^{B}}\left|\overline{\mathbf{h}}_{t, i}^{\dagger} \mathbf{f}_{j, i}\right|$, where $\overline{\mathbf{h}}_{t, i}=\mathbf{h}_{t, i} /\left\|\mathbf{h}_{t, i}\right\|$. The resulting quantization error is related to the chordal distance between the channel, $\overline{\mathbf{h}}_{t, i}$, and the selected codeword, $\hat{\mathbf{f}}_{t, i}$, given by

$$
\mathrm{d}_{\min , t, i}=\sqrt{1-\left|\overline{\mathbf{h}}_{t, i}^{\dagger} \hat{\mathbf{f}}_{t, i}\right|^{2}}
$$

The BS maintains a separate codebook for each user. Once the $\mathrm{BS}$ receives the feedback from all the users, it performs zero-forcing beamforming (ZFBF) [24]. In ZFBF, the BS concatenates all the received codewords from different users into a quantized channel matrix $\hat{\mathbf{H}}_{t}=\left[\hat{\mathbf{f}}_{t, 1}, \hat{\mathbf{f}}_{t, 2}, \ldots, \hat{\mathbf{f}}_{t, K}\right]$ and takes the pseudoinverse of the quantized channel matrix to obtain 
a beamforming weight matrix $\mathbf{W}_{t}=\hat{\mathbf{H}}_{t}\left(\hat{\mathbf{H}}_{t}^{\dagger} \hat{\mathbf{H}}_{t}\right)^{-1}$. The precoding vector for the $i^{\text {th }}$ user is the normalized $i^{\text {th }}$ column of the beamforming weight matrix, $\mathbf{p}_{t, i}=\mathbf{W}_{t}(:, i) /\left\|\mathbf{W}_{t}(:, i)\right\|$. Now, we can define the transmitted signal from the BS as $\mathbf{x}_{t}=\sum_{i=1}^{K} \mathbf{p}_{t, i} \mathbf{s}_{t, i}$, where $\mathbf{s}_{t, i}$ is a data symbol for the user $i$. The sum-rate for the MU MISO system described above can be written as

$$
\mathrm{C}_{t, \text { sum }}=\sum_{i=1}^{K} \log _{2}\left(1+\frac{\frac{\rho}{K}\left|\mathbf{h}_{t, i}^{\dagger} \mathbf{p}_{t, i}\right|^{2}}{1+\frac{\rho}{K} \sum_{k=1, k \neq i}^{K}\left|\mathbf{h}_{t, i}^{\dagger} \mathbf{p}_{t, k}\right|^{2}}\right) .
$$

In codebook-based precoding, the imperfect CSI at the BS introduces multiuser interference leading to a capacity loss. When the channel is highly correlated both temporally and spatially, then the quantization errors of the codebook can be reduced by bringing the codewords close to each other. Also, the incremental change in the position of codewords after each feedback helps differential codebooks to follow the channel.

\section{Differential Codebook Design And Procedure}

In this section, we explain the proposed differential codebook generation method and procedure in a temporally and spatially correlated channel. The proposed differential codebook design is a modified version of the Grassmannian codebook and to modify it according to the channel, scaling and rotation techniques are required (see Sec. IV). The differential codebook generation and feedback steps are as follows:

- Consider a rank-1 Grassmannian codebook denoted by $\mathbf{F}_{G, i}$.

- Define a vertex as, $\mathbf{C}=[1,0, \ldots 0]^{T}$, also known as the basis vector.

- Modify the Grassmannian codebook, $\mathbf{F}_{G, i}$, such that its center is at $\mathbf{C}$. This step involves selecting the Grassmannian codeword that is closest to the basis vector $\mathbf{C}$ and then computing the rotation matrix between them. Finally, rotate all the Grassmannian codebook entries using this rotation matrix.

- Now scale the rotated codewords by a factor $\alpha$, where $\alpha$ is a scaling parameter that defines the radius of the differential codebook. This step completes the generation of the differential codebook denoted by $\mathbf{F}_{\mathrm{diff}, i}$.

- At time $t=0$ (time is represented in multiples of the feedback interval), the user $i$ selects the nearest codeword $\hat{\mathbf{f}}_{0, i}$ from the fixed or base codebook, $\mathbf{F}_{i}$, (e.g 3GPP LTE rel.8 or RVQ) and feeds back the index of the selected codeword, $\hat{\mathrm{f}}_{0, i}$, to the BS.

- Before the next feedback, both BS and user $i$ rotate their differential codebooks such that the selected codeword at time $t=0$ (from the base codebook) becomes the new center of the differential codebook for the next feedback at time $t=1$, and the process continues.

- The differential codebook is reset after every $N$ feedback intervals [20].
The differential codebook feedback approach for time $t=1$ and $t=2$ is depicted in Fig. 1 and Fig. 2 respectively.

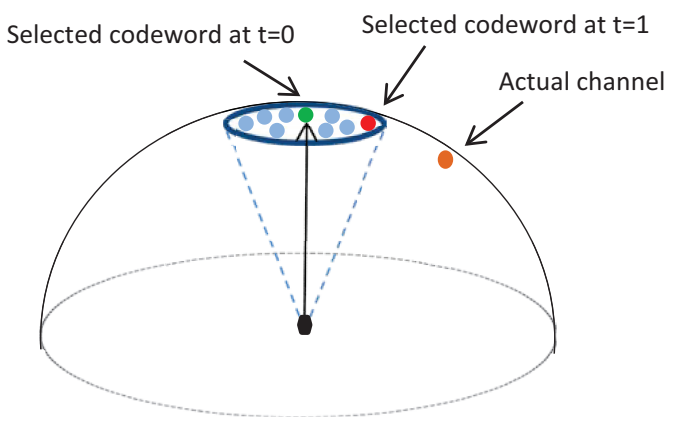

Fig. 1. Structure of the differential codebook at time, $t=1$.

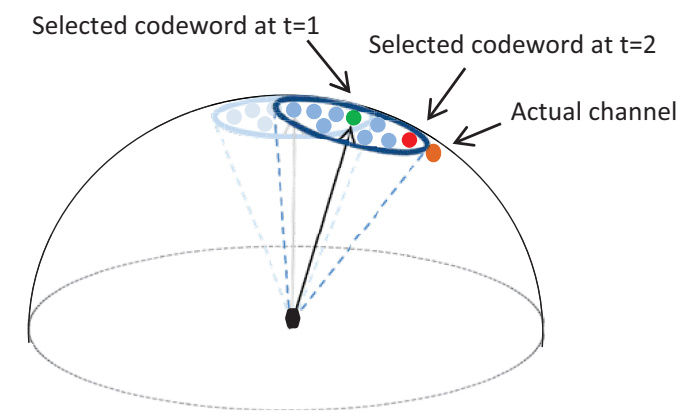

Fig. 2. Structure of the differential codebook at time, $t=2$.

\section{Operations on Differential Codebook}

In this section, we explain the SVD-based rotation method along with both fixed and adaptive scaling techniques. We perform these operations on the Grassmannian codebook to generate the differential codebook.

\section{A. Rotation Technique}

We introduce an SVD-based ${ }^{2}$ rotation operation that can effectively rotate the previously selected codeword $\hat{\mathbf{f}}_{t-1, i}$ to the current selected codeword $\hat{\mathbf{f}}_{t, i}$. To rotate a codeword, we first perform SVD operation on the codewords, given by

$$
\operatorname{SVD}\left(\hat{\mathbf{f}}_{t, i} \hat{\mathbf{f}}_{t-1, i}^{\dagger}\right)=\underbrace{\left[\begin{array}{ll}
\hat{\mathbf{f}}_{t, i} & \hat{\mathbf{f}}_{t, i}^{\perp}
\end{array}\right]}_{\mathbf{U}} \mathbf{D} \underbrace{\left[\begin{array}{ll}
\hat{\mathbf{f}}_{t-1, i} & \hat{\mathbf{f}}_{t-1, i}^{\perp}
\end{array}\right]^{\dagger}}_{\mathbf{V}^{\dagger}},
$$

where, $\hat{\mathbf{f}}_{t-1, i}^{\perp}$ and $\hat{\mathbf{f}}_{t, i}^{\perp}$ denote the null space of the previouly selected codeword and the current selected codeword respectively. A diagonal matrix with zero non-diagonal entries containing the singular values of $\hat{\mathbf{f}}_{t, i} \hat{\mathbf{f}}_{t-1, i}^{\dagger}$ is denoted by D. Now, we can define the rotation matrix, $\Theta_{t, i}$, as the

\footnotetext{
${ }^{2}$ The advantage of SVD-based rotation is that it can not only rotate a singlestream codebook but also a multi-stream codebook. This is not discussed here due to limited space.
} 
multiplication of left and right unitary matrices $\mathbf{U}$ and $\mathbf{V}^{\dagger}$, given by $\boldsymbol{\Theta}_{t, i}=\mathbf{U V}^{\dagger}$. This rotation matrix, $\boldsymbol{\Theta}_{t, i}$, is a unitary matrix with determinant equal to one.

\section{B. Scaling Technique}

After rotating the codewords of the Grassmannian codebook, $\mathbf{F}_{G, i}$, such that the basis vector, $\mathbf{C}$, becomes the new center for the modified codebook (as explained in Sec. III), the scaling operation is performed. In this work, we consider the scaling method presented in [15]. Each codeword is scaled by a factor $\alpha$ independently such that for the first codeword $\mathbf{f}_{1, i}$, the scaling is given by

$$
\mathbf{s}\left(\mathbf{f}_{1, i}\right)=\left[\begin{array}{c}
\sqrt{\frac{1-\alpha^{2}\left(1-\left|\mathrm{f}_{1, i, 1}\right|^{2}\right)}{\left|\mathrm{f}_{1, i, 1}\right|^{2}}} \mathrm{f}_{1, i, 1} \\
\alpha \mathrm{f}_{1, i, 2} \\
\vdots \\
\alpha \mathrm{f}_{1, i, n_{\mathrm{T}}}
\end{array}\right],
$$

where $\mathrm{f}_{1, i, l}, l=1, \ldots, n_{\mathrm{T}}$ are the column entries of the codeword, $\mathbf{f}_{1, i}$. This scaling technique affects the norm of the codewords. Therefore, we divide the scaled codeword by its norm, in order to maintain a unit norm. Hence,

$$
\tilde{\mathbf{s}}\left(\hat{\mathbf{f}}_{1, i}\right)=\frac{\mathbf{s}\left(\hat{\mathbf{f}}_{1, i}\right)}{\left\|\mathbf{s}\left(\hat{\mathbf{f}}_{1, i}\right)\right\|} .
$$

\section{Adaptive Scaling Technique}

An adaptive scaling technique for the differential codebook is presented in this section, where after each feedback the codebook is re-scaled using (9). We denote the adaptive scaling parameter by $\tilde{\alpha}$. The adaptive scaling parameter depends upon the current precoding and previous precoding vectors such that

$$
\tilde{\alpha}_{t+1, i}=\sqrt{1-\left|\hat{\mathbf{f}}_{t-1, i}^{\dagger} \hat{\mathbf{f}}_{t, i}\right|^{2}},
$$

where $\tilde{\alpha}_{t+1, i}$ is a new scaling parameter for the user $i$. If the precoding vectors are same for time $t-1$ and $t$, then the differential codebook will keep the previous scaling factor, $\tilde{\alpha}_{t, i}$. This method is effective when the differential codebook reset time is small and the user is moving with slow speed. The adaptive scaling technique is also discussed in [20], where both $\mathrm{BS}$ and user are required to share the additional information of the time correlation. In the same manner as [20], this adaptive scaling technique keeps shrinking the codebook after every feedback time, this is why reset is necessary ${ }^{3}$.

\section{Simulation Results}

We perform Monte Carlo simulations in order to evaluate the capacity performance of the MU MISO system with the proposed differential codebook. The differential codebook design is based on the 4 bit rank-1 Grassmannian codebook. The feedback interval is $5 \mathrm{~ms}$ and we assume that the feedback link is lossless with zero delay. The differential codebook reset time is $N=8$ feedback intervals (i.e $40 \mathrm{~ms}$ ). The number of transmit antennas at the $\mathrm{BS}$ is four $n_{\mathrm{T}}=4$ and each user

\footnotetext{
${ }^{3}$ In our journal work, the codebook will be made resilient to errors that occur when the reset period is large.
}

is equipped with one receive antenna $n_{R}=1$. The carrier frequency is $2.5 \mathrm{GHz}$.

\section{A. Temporally Correlated Channels}

In this subsection, we present the results for the temporally correlated channel generated using the model given in (2) with no spatial correlation. The base codebook that each user uses at time $t=0$ is a 4 bit RVQ [21] codebook. We compare the proposed differential codebook performance with the polar-cap differential codebook [20] and the rotationbased differential codebook method 1 [18]. We evaluate the performance of the MU MISO system with both fixed scaling, $\alpha$, and adaptive scaling, $\tilde{\alpha}$. The fixed scaling parameter, $\alpha$, is same as that used in [20] for different user speeds. In Figs. 3 and 4, sum-rate versus time results are shown for $\mathrm{v}$ $=1 \mathrm{~km} / \mathrm{h}$ and $\mathrm{v}=5 \mathrm{~km} / \mathrm{h}$ respectively. These figures show that the proposed differential codebook outperforms the polarcap and rotation-based codebooks in a temporally correlated channel. This is due to the fact that in the proposed differential codebook, unlike [20], the codewords are evenly distributed inside the radius, $\alpha$, hence reducing quantization errors, once the differential codebook starts following the channel. The capacity loss is larger when the speed is $v=5 \mathrm{~km} / \mathrm{h}$ in Fig. 4 as compared to Fig. 3 where the speed is $v=1 \mathrm{~km} / \mathrm{h}$. As the user speed increases, the minimum distance $d_{\text {min }}$ between the channel and the selected codeword also increases, which is the reason for the large capacity loss. In Fig. 4, the sum-rate performance with adaptive scaling starts to degrade as time increases, emphasizing the need to reset the process.

\section{B. Spatially and Temporally Correlated Channels}

The spatially and temporally correlated channel is modeled using (3). We use the 4 bit 3 GPP LTE rel. 8 codebook $^{4}$ as a base codebook in the spatially and temporally correlated channel because the DFT codewords present in the LTE codebook improve the performance in the spatially correlated channel [20]. We evaluate the performance of the proposed differential codebook with fixed scaling, $\alpha$, and compare it to the polarcap differential codebook [20]. The sum-rate performance over time for the proposed differential codebook is presented in Fig. 5 with $\mathrm{v}=1 \mathrm{~km} / \mathrm{h}$ and $|r|=0.95$. The scaling parameter is small $(\alpha=0.1142)$ because in high spatial correlation scenarios, the channel variation is strongly focussed towards the direction of transmit correlation. The proposed differential codebook outperforms the polar-cap differential codebook. Similarly, in Fig. 6, the performance of the proposed differential codebook is shown for $\mathrm{v}=3 \mathrm{~km} / \mathrm{h}$ and $\mathrm{v}=1 \mathrm{~km} / \mathrm{h}$ with $|r|$ $=0.95$ and $|r|=0.5$ respectively. It is shown that the proposed differential codebook performs well for all ranges of spatial correlation. In Fig. 7, the sum-rate performance is evaluated when $\mathrm{v}=3 \mathrm{~km} / \mathrm{h}$ and $|r|=0.5$. The performance is analyzed with two scaling parameters; 0.1142 and 0.2836 . As the spatial

\footnotetext{
${ }^{4}$ Scheduling the users is not part of this paper, therefore, during simulation we discard the case when more than one user selects the same codeword from the LTE codebook. Reporting the same codeword affects the channel inversion process of $\mathrm{ZFBF}$ scheme.
} 


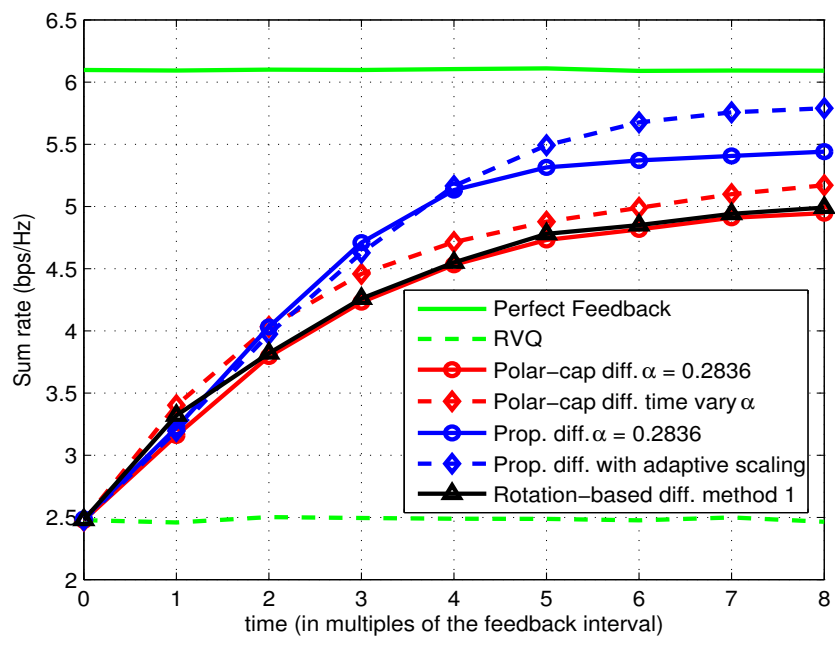

Fig. 3. Sum-rate vs. time in a temporally correlated channel at $\mathrm{SNR}=10 \mathrm{~dB}$ with $\mathrm{v}=1 \mathrm{~km} / \mathrm{h}(\epsilon=0.9987)$

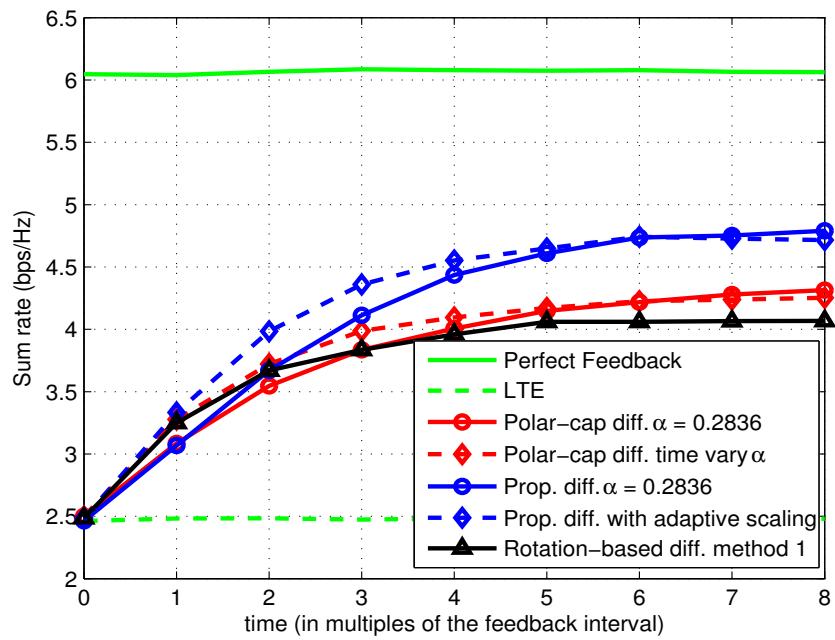

Fig. 4. Sum-rate vs. time in a temporally correlated channel at $\mathrm{SNR}=10 \mathrm{~dB}$ with $\mathrm{v}=5 \mathrm{~km} / \mathrm{h}(\epsilon=0.9672)$

correlation decreases, the channel approaches i.i.d., thus, the scaling parameter, $\alpha$, needs to be increased. This is the reason that the performance gain with $\alpha=0.2836$ is higher than $\alpha=$ 0.1142 .

Figure 8 shows the average minimum chordal distance, $\mathbb{E}\left(\mathrm{d}_{\min }\right)$, where $\mathrm{d}_{\min }$ is given by (5) between the selected codebook entry and the actual channel for different user speeds in temporally correlated channels. It is seen that small values of the scaling parameter, $\alpha$, yield a small minimum chordal distance when the user speed is slow. As the speed increases, large scaling parameters perform better by yielding small average $d_{\min }$ values. It is also evident that a scaling parameter of 0.1 is too small and does not help the differential codebook to track the temporally correlated channel. A suitable choice of $\alpha$ for different speeds can be obtained from Fig. 8, and is

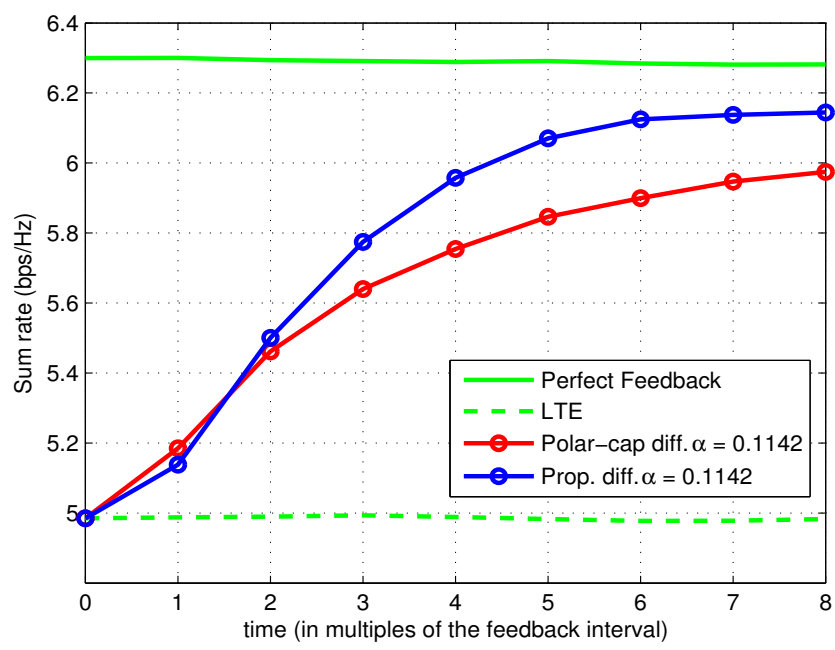

Fig. 5. Sum-rate vs. time in a spatially/temporally correlated channel at $\mathrm{SNR}=10 \mathrm{~dB}$ with $\mathrm{v}=1 \mathrm{~km} / \mathrm{h}(\epsilon=0.9987)$ and $|r|=0.95$.

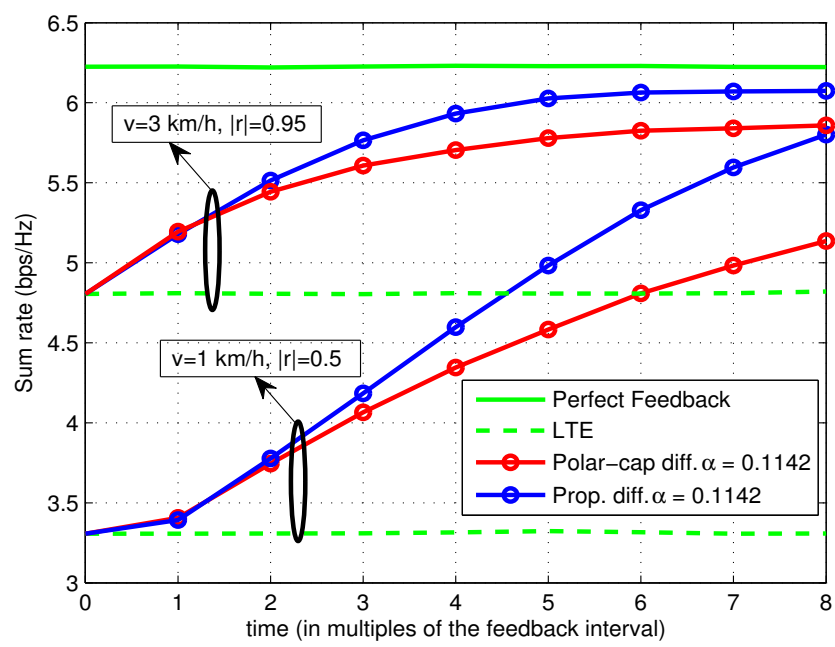

Fig. 6. Sum-rate vs. time in a spatially/temporally correlated channel at $\mathrm{SNR}=10 \mathrm{~dB}$.

also given in Table I.

TABLE I

SCAling Parameters in Temporally CorRelated Channels

\begin{tabular}{|c||c|c|c|c|}
\hline User speed v $(\mathrm{km} / \mathrm{h})$ & $2-6$ & $6-12$ & $12-17$ & $17-20$ \\
\hline \hline Scaling parameter $\alpha$ & 0.3 & 0.5 & 0.7 & 0.9 \\
\hline
\end{tabular}

It is evident from the simulation results that the proposed differential codebook successfully exploits the correlation, both spatial and temporal, present in the channel and helps the system to achieve a better sum-rate performance.

\section{CONCLUSION}

In this paper, we propose a differential codebook design that is a modified version of the Grassmannian codebook. The 


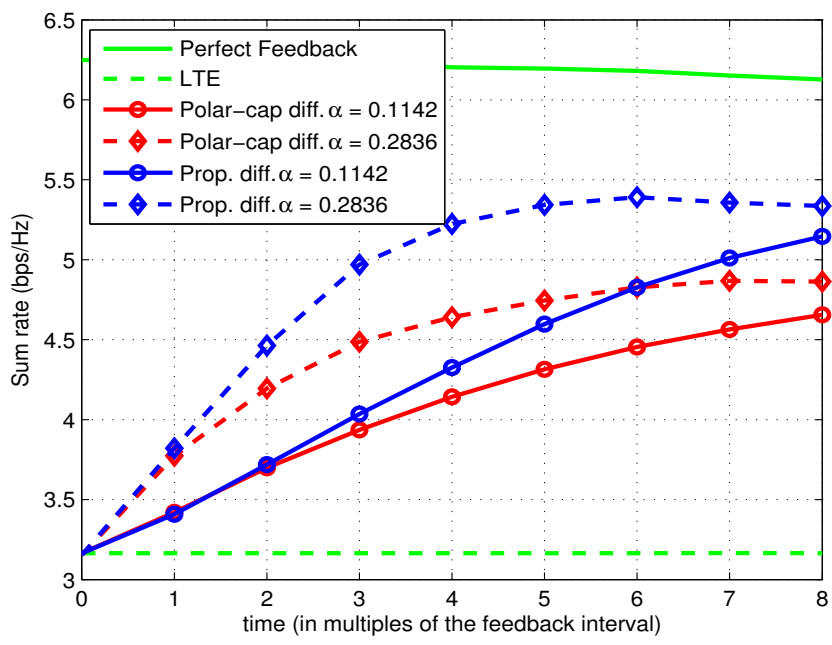

Fig. 7. Sum-rate vs. time in a spatially/temporally correlated channel at $\mathrm{SNR}=10 \mathrm{~dB}$ with $\mathrm{v}=3 \mathrm{~km} / \mathrm{h}(\epsilon=0.9881)$ and $|r|=0.5$.

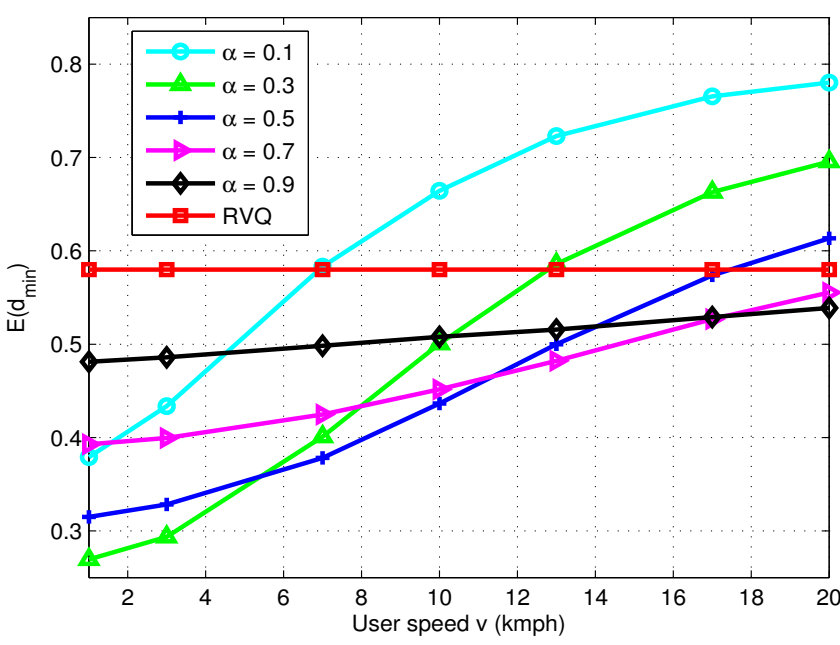

Fig. 8. Average minimum chordal distance vs. user speed in temporally correlated channels.

proposed differential codebook performs well under spatially and temporally correlated channels. We show via simulations that the proposed differential codebook outperforms the polarcap differential codebook structure [20] that has most of the codewords on the radius of the polar-cap. In addition to this, we also introduce an SVD-based rotation method that effectively rotates the codewords. When the differential codebook reset time is small, the adaptive scaling technique is proposed that can further improve the sum-rate performance of the users moving with slow speeds. The optimization of the scaling parameter for different user speeds is left to future work. In this paper, the main focus was to improve the capacity performance while keeping the feedback overhead the same.

\section{REFERENCES}

[1] D. J. Love, R. W. Heath Jr., W. Santipach, and M. L. Honig, "What is the value of limited feedback for MIMO channels?" IEEE Commun. Mag., vol. 42, pp. 54 - 59, Oct. 2004.

[2] D. J. Love, R. W. Heath Jr., and T. Strohmer, "Grassmannian beamforming for multiple-input multiple-output wireless systems," IEEE Trans. Inf. Theory, vol. 49, pp. 2735 - 2747, Oct. 2003.

[3] D. J. Love, R. W. Heath Jr., V. K. N. Lau, D. Gesbert, B. D Rao, and M. Andrews, "An overview of limited feedback in wireless communication systems," IEEE J. Sel. Areas Commun., vol. 26, pp. 1341 - 1365, Oct. 2008.

[4] D. J. Love and R. W. Heath Jr., "Limited feedback diversity techniques for correlated channels," IEEE Trans. Veh. Technol., vol. 55, pp. 718 722, March 2006

[5] M. Zhang, M. Shafi, P. J. Smith, and P. A. Dmochowski, "Precoding performance with codebook feedback in a MIMO-OFDM system," in Proc. IEEE International Conference on Communications (ICC), pp. 1 $-6,2011$.

[6] IEEE 802.16m-09/0034r4, IEEE 802.16m System Description Document $(S D D)$. IEEE, Dec. 2010

[7] B. Clerckx, D. Mazzarese, G. Kim, and S. Kim, "Multiuser MIMO downlink made practical: Application to IEEE $802.16 \mathrm{~m}$," in Proc. IEEE Vehicular Technology Conference (VTC), pp. 1 - 5, 2009.

[8] Q. Li, G. Li, W. Lee, M. il Lee, D. Mazzarese, B. Clerckx, and Z. Li, "MIMO techniques in WiMAX and LTE: a feature overview," IEEE Commun. Mag., vol. 48, pp. 86 - 92, May 2010.

[9] NTT DoCoMo, Telefonica, Sprint, T-mobile, Telecom Italia, Orange, Verizon Wireless, Vodafone, "LTE performance review and way forward," 3GPP, R1-072543, May, 2007.

[10] Samsung, ZTE, ETRI, ITRI, ASUStek, CHTTL, "Way forward on SU MIMO codebook design for 4 Tx dual polarised antennas," $3 G P P, R 1$ 072582, May, 2007.

[11] Samsung, "MIMO for long term evolution," 3GPP, R1-050889, Feb., 2007.

[12] Texas Instruments, Motorola, NTT DoCoMo, NEC, "Proposed way forward on codebook design for E UTRA," 3GPP, R1-070728, Feb., 2007.

[13] Free scale semiconductor, "Overview and way forward on MIMO pre coding in DL EUTRA," 3GPP, R1-070220, Jan., 2007.

[14] Samsung, "Way forward on codebook for SU MIMO precoding," 3GPP, R1-073181, Jan., 2007.

[15] V. Raghavan, R. W. Heath Jr., and A. V. Sayeed M., "Systematic codebook designs for quantized beamforming in correlated MIMO channels," IEEE J. Sel. Areas Commun., vol. 25, pp. 1298 - 1310, Sep. 2007.

[16] T. Abe and G. Bauch, "Differential codebook MIMO precoding technique," in Proc. IEEE Global Telecommunications Conference (Globecom'07), pp. 3963 - 3968, 2007.

[17] T. Kim, D. J. Love, B. Clerckx, and S. J. Kim, "Differential rotation feedback MIMO system for temporally correlated channels," in Proc. IEEE Global Telecommunications Conference (Globecom'08), pp. 1 5, 2008.

[18] T. Kim, D. J. Love, and B. Clerckx, "MIMO systems with limited rate differential feedback in slowly varying channels," IEEE Trans. Commun., vol. 59, pp. 1175 - 1189, April 2011.

[19] IEEE C802.16m-09/1530r3, Improved Differential Codebooks for IEEE 802.16m Amendment Working Document. IEEE, July 2009.

[20] J. Choi, B. Clerckx, N. Lee, and G. Kim, "A new design of polar-cap differential codebook for temporally/spatially correlated MISO channels," IEEE Trans. Wireless Commun., vol. 11, pp. 703 -711, Feb. 2012.

[21] C. K. Au-Yeung and D. J. Love, "On the performance of random vector quantization limited feedback beamforming in a MISO system," IEEE Trans. Wireless Commun., vol. 6, no. 2, pp. 458 - 462, 2007.

[22] J. Proakis, Digital Communications. 4th Edition, McGraw Hill, 2000.

[23] B. Clerckx, G. Kim, and S. Kim, "Correlated fading in broadcast MIMO channels: Curse or blessing?," in Proc. IEEE Global Telecommunications Conference (Globecom'08), pp. 1 - 5, 2008.

[24] T. Yoo and A. Goldsmith, "On the optimality of multiantenna broadcast scheduling using zero-forcing beamforming," IEEE J. Sel. Areas in Commun., vol. 24, pp. 528 - 541, March 2006. 